\title{
MedienPädagogik
}

Zeitschrift für Theorie und Praxis der Medienbildung

www.medienpaed.com

ISSN 1424-3636

Jahrbuch Medienpädagogik 15:

Erziehungswissenschaftliche und medienpädagogische Online-Forschung:

Herausforderungen und Perspektiven

Herausgegeben von Johannes Fromme, Stefan Iske, Therese Leik, Steffi

Rehfeld, Jasmin Bastian, Manuela Pietraß und Klaus Rummler

\section{Offline- und Online-Umgebungen als Kontexte integrierter Forschungsdesigns}

\author{
Johannes Wahl und Sebastian Zimmer
}

\section{Zusammenfassung}

Die zunehmende Nutzung von Online-Kommunikationskanälen vereinfacht nicht nur den alltäglichen, zwischenmenschlichen Austausch, sondern eröffnet auch der erziehungswissenschaftlichen Forschung neue Möglichkeiten. Gleichzeitig stehen Chancen wie der Reichweitenerhöhung von Forschungsaktivitäten auch Herausforderungen bspw. im Bereich der Validität gegenüber. Vor diesem Hintergrund geht der Beitrag der Frage nach, ob sich diese Nachteile durch die methodologisch fundierte Kombination von Offline- und Online-Umgebungen kompensieren lassen. Anhand eines Forschungsszenarios werden drei verschiedene Designs konzipiert, die auf genau diese Herausforderung eingehen. Dazu wird eine Mixed Methods Perspektive eingenommen, um verschiedene Möglichkeiten aufzuzeigen, die einzelne Schwächen der Methoden adäquat ausgleichen oder sogar Synergieeffekte erzielen. 


\title{
Offline and Online Environments as Fields of Integrated Research Designs
}

\begin{abstract}
The increasing usage of online communication channels is not only simplifying the everyday interpersonal exchange of information, but also opens up new possibilities for the educational science research. Concurrently, the chances of increasing reach of research activities are in opposition to challenges, e.g. the field of validity. For these reasons, the article follows the questions whether these disadvantages can be compensated by methodologically rigorous combinations of offline and online environments. Based on a potential research scenario, three different research designs are devised to exactly address the aforementioned challenges. Thereto, a mixed methods perspective is used to display several possibilities that appropriately compensate specific weaknesses of the empirical methods or even achieve synergy effects.
\end{abstract}

\section{Menschliche Kommunikation im Kontext von Di- gitalität}

Fragt man nach den Stärken und Schwächen von Methoden aus dem Kanon der empirischen Sozialforschung, so lassen sich bspw. Beurteilungskriterien anführen, die auf grundlegende Qualitätskriterien wie die Gegenstandsangemessenheit (Przyborski und Wohlrab-Sahr 2014) oder den Informationsgehalt der generierten Daten (Döring und Bortz 2016) verweisen. Ein weiterer, im Vergleich zu klassischen Gütekriterien weniger stark fokussierter Aspekt bilden Einflussgrössen, die mit der (un)mittelbaren Interaktion zwischen Forschenden und Beforschten zusammenhängen. Betrachtet man diese aus einer kommunikationstheoretischen Perspektive, so basieren diese Effekte auf der grundlegenden Funktion von menschlicher Kommunikation: dem interpersonalen Austausch von Informationen, Bedeutungsgehalten und Sinnkonstruktionen (Pürer, Springer und 
Eichhorn 2015). ${ }^{1}$ Bei diesem Austausch kann es bspw. aufgrund von Interpretationsleistungen der Akteurinnen und Akteure zum Informationsverlust bzw. zu Akzentverschiebungen kommen. Dabei lässt sich in Anlehnung an Schütz (1971) ein Spektrum zwischen umweltlicher, mitweltlicher und vorweltlicher Kommunikation erzeugen. Die umweltliche Kommunikation beschreibt dabei eine direkte Kommunikation mit einer Vielzahl redundanter Informationskanäle, die eine sich gegenseitige Verifizierung der übertragenen Informationen von Sendenden an Empfangende und damit eine eindeutige Interpretation zulässt. Mit der Abnahme redundanter Kanäle bei gleichzeitiger Zunahme von Zeitverzögerung der Zustellung der Information wird die Kommunikation zunehmend mitweltlicher. Findet sie schliesslich nur noch einseitig statt, z.B. durch das Lesen von Blogposts längst Verstorbener, dann ist die Kommunikation am Endpunkt angekommen und damit vorweltlich. In Kombination mit dem Shannon-Weaver-Modell von Kommunikation (Weaver und Shannon 1949) bzw. dem von Shannon (1948) definierten Rauschen, welches zunimmt, je höher die Interpretationsleistung der Empfangenden ist, lässt sich sehr gut beschreiben, dass eine E-Mail eine vergleichsweise hohe Interpretationsleistung (mitweltliches Rauschen) bedingt, da ihre Inhalte häufig auf Wesentliches reduziert sind, sodass redundante Verifikatoren fehlen. Die Avatarisierung, hier im vereinfachten Sinne des digitalen Abbilds, versucht dem mit Emoji, Animoji und Memoji ein Stück weit entgegenzuwirken, birgt aber auch die Möglichkeit des bewussten Täuschens, wenn ein positiv konnotierter Emoji hinter eine negative Nachricht gesetzt wird und vice versa. Diese Phänomene zeigen sich nicht nur in alltäglichen Gesprächssituationen, sondern spielen auch in Forschungskontexten eine wichtige Rolle.

Die Quantität und Qualität des Informationsflusses von Sendenden zu Empfangenden variieren im Kommunikationsprozess demzufolge aufgrund der verwendeten Medien. Folgt man der klassischen

1 An dieser Stelle sei auf den terminologischen Unterschied zwischen Interaktion (Fokus auf das spezifische interpersonale Beziehungsgefüge) und Kommunikation (Fokus auf den prozessualen Austausch von Informationen) verwiesen (Pürer, Springer und Eichhorn 2015). 
Definition von Burkart (2002), so bilden Medien den mehr oder weniger komplexen Vermittlungskontext der Informationsübertragung. Durch diese relativ weite Definition von Medien lassen sich sowohl die menschliche Gestik, Mimik und Sprache im Sinn von primären Medien einordnen als auch online-basierte Regelstrukturen im Sinn von quartären Medien klassifizieren (Burkart 2002) und miteinander vergleichen. Kommunikation vollzieht sich aus dieser Perspektive heraus in allen Fällen medial vermittelt. Erst die Frage, inwiefern in diesen Prozessen auch technische Geräte zum Einsatz kommen, also auf welche Weise der Informationsaustausch technisch vermittelt wird, eröffnet eine medienpädagogisch unmittelbar anschlussfähige Perspektive auf menschliche Kommunikation. So lassen sich bspw. die Wechselwirkungen von Social Media-Nutzung und der Kommunikationsfrequenz bzw. -dauer in unterschiedlichen Bevölkerungsgruppen oder die Nutzenerwartungen des kontrollierten Informationsaustauschs durch Phänomene der Avatarisierung erforschen. Gerade durch die technischen Entwicklungen und die damit korrespondierende Mediatisierung sind in den letzten Jahren vielfältige Varianten menschlicher Kommunikation entstanden, die das Spektrum exklusiv analoger Kommunikationsumgebungen massiv erweitern (Kammerl 2018).

Die oben genannten Beispiele stehen exemplarisch für eine Vielzahl an Kommunikationsformaten, die aufgrund ihrer strukturellen Kopplung mit online-basierten Geräten mit dem Begriff der Digitalität verbunden werden. Durch diesen wird das Verhältnis von technologischen Entwicklungen und gesellschaftlichen Strukturen in den Mittelpunkt gerückt. Im Sinn einer Statusbeschreibung und weniger eines Wandlungsprozesses wird die Vernetzung von analogen und digitalen Wirklichkeiten als lebensweltliche Konstante beschrieben und pointiert als «Kultur der realen Virtualität» (Castells 2017, 459) greifbar. Doch auch wenn damit die Differenz zwischen analogen und digitalen Umwelten graduell eingeebnet wird, bleibt sie im Grundsatz bestehen. 
In Bezug auf die bereits im Titel des Beitrags angeschnittene heuristische Differenz zwischen Offline- und Online-Umgebungen lässt sich die im Begriff der Digitalität angelegte Verschränkung von analogen und digitalen Kontexten nutzen. Dementsprechend werden im vorliegenden Beitrag in Anlehnung an Pietraß (2018) einerseits analoge bzw. Offline-Umgebungen wie bspw. alltägliche Gesprächssituationen in leiblicher Anwesenheit und andererseits digitale bzw. Online-Umgebungen wie Social Media analytisch differenziert. Neben dieser inhaltlichen Logik ist die Trennung in Offline- und Online-Umgebungen geboten, um die im weiteren Verlauf darzustellenden Konstruktionsmöglichkeiten von Forschungsdesigns möglichst trennscharf herauszuarbeiten.

\section{Erforschung sozialer Phänomene im Kontext von Digitalität}

Wie bereits im vorigen Kapitel kurz angedeutet, ergeben sich in einer mediatisierten und von Digitalität geprägten Gesellschaft vielfältige Kommunikationsoptionen, die sich auch für die medienpädagogische Theoriebildung erforschen lassen. Die Grundlage für diesen Prozess bilden Forschungsergebnisse, die durch unterschiedliche method(olog)ische Zugänge der empirischen Sozialforschung generiert werden können. Sie beschäftigt sich mit der «Beschreibung, Erklärung, Vorhersage und Veränderung» (Döring und Bortz 2016, 5) sozialer Phänomene wie den oben beschriebenen und hält ein breites Spektrum an Methoden bereit, um vergangene, aktuelle und begrenzt auch zukünftig eintretende Entwicklungen zu erforschen.

Genauso wie sich soziale Konstellationen verändern, sind auch die Methoden der empirischen Sozialforschung von Wandlungsbzw. Innovationsprozessen betroffen, was sich exemplarisch anhand der Entwicklung der Online-Forschung nachzeichnen lässt (Welker 2014). Eine offensichtliche Entwicklung besteht in der Nutzung neuer technischer Komponenten für Forschungsaktivitäten 
in Offline-Umgebungen: So werden bspw. seit mehreren Jahren schriftliche Befragungen online-basiert durchgeführt, wobei das einzige Differenzmerkmal zu den analogen Varianten in der Nutzung des Internets besteht (Wagner und Hering 2014). In diesem und in weiteren Zusammenhängen bilden methodische Zugänge, die auf Online-Umgebungen aufbauen, eine Alternative zur Erforschung sozialer Phänome mittels offline-basierter Methoden.

Die Wahlmöglichkeit zwischen Offline- und Online-Kontexten von empirischer Sozialforschung existiert jedoch nicht genuin. Gerade für die effektive Auswertung grosser Datensätze im Sinn des Datamining oder für die Analyse spezifischer Datensorten wie Logfiles kommen keine Methoden zur Anwendung, die auf OfflineUmgebungen aufbauen (Zeller 2017), sodass in solchen Fällen ausschliesslich Online-Kontexte als gegenstandsangemessen zu bewerten sind. Die Limitierung offline-basierter Zugänge zum jeweiligen Forschungsgegenstand zeigt sich auch in einer anderen Hinsicht. Durch die leibliche Anwesenheit der Forschenden im Forschungsfeld treten zwangsläufig Validitätsbedrohungen auf, die sich auf den Forschungsverlauf und dessen Resultate auswirken. Diese werden bspw. im Rahmen der Umfrageforschung als Interviewereffekte aufgegriffen (Glantz und Michael 2014) oder auch in der ethnografischen Forschung über die Rolle der Forschenden im Feld thematisiert (Przyborski und Wohlrab-Sahr 2014). Diese exemplarisch angeführte Begrenzung kann zu den bereits am Anfang des Artikels beschriebenen Phänomenen des Informationsverlustes durch Interpretationsleistungen und der Akzentverschiebung im Rahmen zwischenmenschlicher Kommunikation führen.

Um diesen problematischen Konstellationen innerhalb von Forschung in Offline-Umgebungen zu begegnen, lassen sich unterschiedliche Strategien einsetzen. In den beiden oben genannten Beispielen der Umfrageforschung und der ethnografischen Forschung bestehen diese bspw. in der prophylaktischen Schulung der Interviewenden und der systematischen Reflexion der eigenen Rolle als 
Forschende im Forschungsprozess. Jenseits dieser Möglichkeiten möchten wir in diesem Artikel eine weitere Möglichkeit diskutieren, um der Problematik zu begegnen. Dazu setzen wir auf der Ebene des Forschungsdesigns an und schlagen vor, offline- und online-basierte Methoden zu integrierten Designs zusammenzufassen. Durch den dazu notwendigen Bezug zu Mixed Method Research (MMR) wird es möglich, die Vorteile von Offline- und Online-Umgebungen zu kombinieren.

\section{Mixed Method Research zur Steigerung der For- schungsgüte}

Durch die zunehmende Rechenleistung von Computern und der insbesondere durch das Internet erweiterten Verfügbarkeit von Daten, haben sich in den letzten Jahrzehnten verschiedene Formate der methodenpluralen Forschung entwickelt. Bereits in den 1920er und 30er Jahren wurden sogenannte Common Sense Studien verwendet für die Erforschung unbekannter Phänomene wie der Arbeitslosigkeit (Jahoda et al. 1975), allerdings ohne klare Systematisierung der Methodenkombination. Diese Systematisierung erfolgte im deutschen Sprachraum erst in den 1960ern durch die Triangulation, unter der zumeist die Kombination zweier quantitativer Methoden verstanden wird (Kuckartz 2014). Mit der Zunahme von Interdisziplinarität und dem Aufweichen des Paradigmenkriegs zwischen quantitativer und qualitativer Methoden in den 1990ern bei gleichzeitiger Verfügbarkeit von neuen, schnellen Computern und einem Zugang zum Internet entwickelten sich zunehmend methodenplurale Ansätze im Sinne der Multi Methods, in denen verschiedene Methoden zur Beantwortung einer Forschungsfrage verwendet, aber nicht miteinander integriert werden (Maxwell 2013). Diese Leistung vollbringt erst die Systematisierung der MMR. Dahinter verbirgt sich die Idee einer Forschung mit der Intention 
«to thoughtfully and strategically mix or combine qualitative and quantitative research methods, approaches, procedures, concepts, and other paradigm characteristics in a way that produces an overall design with multiple (convergent and divergent) and complementary strengths (broadly viewed) and nonoverlapping weaknesses» (Johnson und Christensen 2014, 64).

Erst 1998 wurden die verschiedenen philosophischen wie methodischen Entwicklungen unter dem Begriff 〈Mixed Methodology〉 vereinheitlicht (Tashakkori und Teddlie 1998). In der anschliessenden Dekade konzentrierten sich die Debatten in den Mixed Methods primär auf zwei Schwerpunkte. Erstens Designkonzeptionen, d.h. die systematische Verknüpfung von Methoden unterschiedlicher paradigmatischer Prägung (qualitativ/quantitativ) (Johnson et al. 2007). Zweitens die Positionierung der Mixed Methods bezüglich der qualitativen und quantitativen Paradigmen bzw. deren ontologisch-epistemologischer Grundlagen (Johnson und Onwuegbuzie 2004). Dabei entwickelten sich, je nach Disziplin, teils unterschiedliche Ansätze, auch weil eine klare Abgrenzung zu den Multi Methods ausblieb bzw. immer noch nicht eindeutig besetzt ist (Baur et al. 2017). Derweil löste sich in den letzten zehn Jahren zunehmend die Fokussierung auf ein reines Methodendesign (Mixed Methods), das pragmatisch zur Forschungsfrage passt, hin zu einer ganzheitlichen Betrachtung der Forschung als einen Mixed-Prozess (Mixed Research) von der Forschungsfrage bis zum Fazit (u.a. Leech und Onwuegbuzie 2009).

Grundsätzlich bieten sich MMR-Studien immer dann an, wenn eine umfassendere Antwort auf die Forschungsfrage gefunden werden sollte oder synergetische Auflösungen von Validitätsbedrohungen bzw. eine Erhöhung der Forschungsgüte erreicht werden sollen, d.h. die Schwächen insbesondere der Validität einer Methode sinnhaft durch die Stärken einer anderen ergänzt wird (Creswell und Plano-Clark 2018). Dabei ist eine Integration der verwendeten Methoden eine wesentliche Komponente, um neben der erweiterten 
Validierung auch erweiterte Ergebnisse zu erzielen, sogenannte Meta-Inferenzen. Plakativ versucht sich die Mixed Methods von den Multi Methods insoweit abzugrenzen, dass aus $1+1$ (Methoden) $=2$ (Ergebnis) ein $1+1=3$ wird (Fetters und Freshwater 2015), wobei im Regelfall eher von $1+1=2,4$ auszugehen ist, im Vergleich zum nicht integriertem Methodendesign immer noch ein Zugewinn, der allerdings mit Mehraufwand durch Integration verbunden ist (Kuckartz 2014).

Bei der Kombination von quantitativen und qualitativen empirischen Methoden auf den konkreten Anwendungsfall der Offline-/ Online-Verknüpfung und der rekursiven Validierung der Ergebnisse gilt es besonderes auf synergetische Effekte zu achten, um z.B. die zuvor genannten Interviewereffekte systematisch zu kompensieren bis zu neutralisieren. Dabei ist es von Vorteil, dass bei genauerer Betrachtung bei vielen empirischen Methoden die Übergänge von quantitativ zu qualitativ sehr fliessend sind (Teddlie und Tashakkori 2009). So lassen sich z.B. in einem Fragebogen offene und geschlossene Fragen kombinieren, sodass man bei gleichzeitiger Online- und Offline-Distribution entsprechende Interviewereffekte konkret prüfen könnte. Für die konkrete thematische Erforschung erscheint die Nutzung verschiedener Methoden allerdings sinnhafter. Konkrete Beispiele werden im nächsten Kapitel erläutert.

Erweiterte Optionen der Verknüpfung bieten sich über die Stichprobenziehung an, da sich auch hier Möglichkeiten der Integration bieten (Onwuegbuzie und Collins 2017). Eine repräsentative OnlineUmfrage könnte am Ende anbieten, sich freiwillig für ein Leitfadeninterview zur Verfügung zu melden. Damit liessen sich gezielt abweichende Fälle hinsichtlich ihrer Beweggründe befragen, um so den gesamten Datensatz verständlicher zu machen. Da der Diskurs um die Möglichkeiten der Stichprobenziehung erst seit wenigen Jahren konsequent geführt wird, ist eine konkrete Systematisierung noch ausbleibend (Akremi 2017). 


\section{Method(olog)ische Konsequenzen und Heraus- forderungen}

Aus den bisher dargelegten Inhalten lässt sich ableiten, dass es durchaus Mittel und Wege gibt, um Validitätsbedrohungen, die durch die zwischenmenschliche Kommunikation im Rahmen von Forschungsprozessen zwangsläufig entstehen, systematisch zu minimieren. Dazu bedarf es eines Forschungsdesigns, das unter Bezugnahme auf das gesamte Methodenspektrum der empirischen Sozialforschung sowohl offline- als auch online-basierte empirische Zugänge zum Forschungsgegenstand zusammenfügt.

Die Integration von Offline- und Online-Umgebungen zur Steigerung der Forschungsgüte lässt sich als durchaus herausfordernde Aufgabe am Beginn des jeweiligen Forschungsprozesses beschreiben. Dass sich die Sorgfalt in diesem Abschnitt lohnt, möchten wir nachfolgend anhand eines konkreten Beispiels demonstrieren.

Den Ausgangspunkt unserer method(olog)ischen Überlegungen bildet wie auch bei allen anderen Forschungsvorhaben üblich das Ziel der Forschung. Im vorliegenden Beispiel geht es darum, die Auswirkungen von Smart Speaker (Nielsen 2018) auf milieuspezifisches Mediennutzungsverhalten zu erforschen und die daraus generierten Erkenntnisse auf den Diskursstrang zur Medienbildung (Jörissen und Marotzki 2009) zu beziehen. Um dieses Ziel erreichen, werden zwei forschungsleitende Fragestellungen gebildet, die es empirisch zu bearbeiten gilt:

1. Welche milieuspezifischen Differenzen zeigen sich bei der Mediennutzungsfrequenz im Fall von Smart Speaker?

2. Wie nutzen unterschiedliche Milieus Smart Speaker in ihren Kommunikationsroutinen? 
Basierend auf dem thematischen Fokus und dessen Zuspitzung durch die zwei forschungsleitenden Fragestellungen werden drei integrierte Forschungsdesigns konstruiert, die sowohl zur adäquaten Analyse des Forschungsgegenstands geeignet sind als auch die methodischen Vorteile von Offline- und Online-Umgebungen verbinden. Gleichzeitig wird es hier auch möglich, qualitative und quantitative Methoden zu relationieren.

Im Rahmen von Forschungsdesign 1 wird das milieuspezifische Mediennutzungsverhalten zunächst dadurch erfasst, dass die Nutzung der Smart Speaker mittels Logfileanalyse getrackt wird. Zu diesem Zweck wird eine spezifische App herangezogen und die darin gespeicherten Daten verteilungstheoretisch untersucht. Auf Basis dieses online-basierten und quantitativen Vorgehens lassen sich typische Nutzungsmuster erkennen, die bspw. auf einen spezifischen Verwendungszweck der Smart Speaker schliessen lassen und/oder schlicht zu bestimmten Tageszeiten auftreten. Diese Erkenntnisse, die sich bspw. durch Subgruppenvergleiche erschliessen lassen, werden anschliessend genutzt, um mit Personen aus der Stichprobe Gruppendiskussionen durchzuführen. Dabei werden solche Probandinnen und Probanden zusammengefasst, die entsprechend der Subgruppen über ähnliche Merkmalsausprägungen verfügen. Diese können im Rahmen der Offline-Umgebung über die zuvor generierten Ergebnisse diskutieren, wodurch diese potentiell bestätigt und/ oder vertieft werden. Somit bietet sich Forschungsdesign 1 in solchen Situationen an, in denen eine vorhandene, integrierte Stichprobe dahingehend tiefgreifender analysiert werden soll, dass die quantitativen Ergebnisse mit qualitativen Erkenntnissen gezielt validiert werden können. Das Mediennutzungsverhalten wird auf diese Weise nicht nur als rein quantitative Benutzung betrachtet, sondern durch eine qualitative Ebene kognitiv erklärt.

Eine weitere Möglichkeit, sich dem oben beschriebenen Forschungsziel zu nähern, zeigt Forschungsdesign 2 auf. In diesem Szenario werden zunächst Online-Interviews mit Expertinnen und 
Experten aus dem informationstechnischen und sprachwissenschaftlichen Bereich geführt. Mit diesem qualitativen Zugang wird es möglich, einen Eindruck über die potentiell milieuspezifischen und steuerungsrelevanten Sprachmuster zu gewinnen, auf deren Basis der Smart Speaker spezifische Operationen ausführt. Nachdem durch diesen Zugang Erkenntnisse generiert und in Hypothesen umgewandelt wurden, können diese im Rahmen einer schriftlichen Befragung in leiblicher Anwesenheit überprüft werden. Mittels eines entsprechenden Instruments lässt sich nicht nur die Zustimmung der Studienteilnehmenden zu den von den Expertinnen und Experten antizipierten Nutzungspräferenzen erforschen. Darüber hinaus ermöglichen Fallvignetten und damit verbundene Rankingaufgaben auch die Bedienbarkeit der Smart Speaker in verschiedenen Alltagssituationen einzuschätzen. Zusammengefasst ermöglicht die Variante Forschungsdesign 2 eine direkte Validierung der aus den Interviews gewonnenen Erkenntnisse und Hypothesen hinsichtlich relevanter Aspekte der Forschungsthematik. Gleichzeitig erlaubt dieses verknüpfte Vorgehen eine retrospektive Validierung der Situationseinschätzung, die von den Expertinnen und Experten vorgenommen wurde.

Am Anfang von Forschungsdesign 3 steht nach vorheriger $\mathrm{Zu}-$ stimmung der Studienteilnehmenden eine teilnehmende Beobachtung der Forschenden in deren Wohnungen. Ziel ist es, in dieser Offline-Umgebung die Einbindung der Smart Speaker in die alltäglichen Routinen der Probandinnen und Probanden zu erfassen. Durch diesen empirischen Zugang lässt sich das konkrete Nutzungsverhalten beobachten und damit neben entsprechenden Präferenzen auch weitere Potentiale hinsichtlich der Bedienbarkeit erschliessen. Auf Basis der protokollierten Offline-Situationen lassen sich Fallvignetten erstellen, die die Grundlage für eine online-basierte Befragung bilden. Im Rahmen dieser Erhebung werden die Fallvignetten als alternative Nutzungsszenarien rekontextualisiert und den Probandinnen und Probanden zur Auswahl vorgestellt. Durch die anschliessende Conjoint-Analyse werden die Präferenzen der 
Studienteilnehmenden hinsichtlich dieser alternativen Angebote ermittelt und potentiell Aussagen über milieuspezifisches Mediennutzungsverhallten generiert. Der spezifische Mehrwert von Forschungsdesign 3 besteht in der Ableitung der Präferenzanalyse aus tatsächlichen Nutzungskontexten. Auf Basis der damit verbundenen Kreuzvalidierung der Beobachtungen und der Conjoint-Präferenzen lässt sich die Übertragbarkeit der Erkenntnisse sehr viel präziser ableiten.

Alle drei vorgestellten Forschungsdesigns eröffnen empirische Zugänge zur milieuspezifischen Nutzung von Smart Speakern, die je nach Erkenntnisinteresse als mehr oder weniger gegenstandsadäquat angesehen werden können. Unabhängig von den Unterschieden zwischen den drei Varianten zeigt sich, dass Validitätsbedrohungen mit integrierten Forschungsdesigns, die sowohl Offline- als auch Online-Umgebungen einschliessen, strukturell minimiert werden. Um dies forschungspraktisch umzusetzen, bedarf es nicht nur des Wissens um die geeigneten Methoden und die Kompetenzen zu ihrer Anwendung. Zusätzlich ist ein solches Vorgehen auch mit einem höheren Aufwand an personellen, zeitlichen und damit insgesamt finanziellen Ressourcen verbunden, wodurch es sich eher für umfangreiche Forschungsprojekte als für Qualifizierungsarbeiten auf studentischem Niveau eignet. Aus forschungsethischer Sicht ist in den jeweiligen Integrationsvarianten zudem das potentielle Spannungsverhältnis zwischen Forschungsinteressen und Rechten der Probandinnen und Probanden zu beachten. Gemäss den Anforderungen zum Schutz der Probandinnen und Probanden sowie der Vertraulichkeit der erhobenen Daten (RatSWD 2017) gilt es vor allem, die genutzten Offline- wie Online-Tools so zu kontrollieren, dass die generierten Daten zu keinem Zeitpunkt des Forschungsprozesses an Dritte weitergegeben werden. 


\section{5. $\quad$ Fazit}

Die im vorherigen Kapitel erläuterten Beispiele erlauben einen kurzen Einblick in die Möglichkeiten, die sich durch die Kombination von Offline- und Online-Umgebungen in einem MMR-Ansatz bieten, wobei in diesem Beitrag insbesondere der Mehrgewinn an Informationen bei gleichzeitiger Reduktion alternativer Erklärungsmodelle bzw. Validitätsbedrohungen in den Blick genommen wurde. Demgegenüber steht ein erhöhter Personal-, Zeit- und Ressourcenaufwand, der vor Beginn eines solchen Forschungsvorhabens einkalkuliert werden sollte. Dies ergibt sich auch aus der Notwendigkeit, dass ein integriertes MMR-Design bereits vor Beginn eines Forschungsprojekts vor allem dahingehend geplant sein muss, die damit einhergehenden Meta-Inferenzen zu beachten und die Reduktion der Validitätsbedrohungen zu erzielen. Der zu erreichende Mehrwert bietet jedoch umfassendere Erkenntnisse über den Forschungsgegenstand. Insbesondere bei neuen Themenfeldern wie den Auswirkungen von Digitalität auf spezifische Zielgruppen ergibt sich die Chance, analog zu den Common Sense Studien der 1920er und 1930er Jahre, mit einer Vielzahl bekannter Methoden das unbekannte Thema sehr viel genauer abstecken zu können.

Die im letzten Kapitel vorgestellten Beispiele der methodischen Verknüpfung legen aber auch gleichzeitig die zweite Ebene der Integration im Forschungsfeld der Digitalität dar: Neben der quantitativ-qualitativen Methodenebene wird in den Beispielen auch der strukturellen Kopplung von analoger und digitaler Umwelt Rechnung getragen. Die spezifische Verwobenheit von Offline- und Online-Aktivitäten in der Lebenswelt, hier im Kontext der Nutzung von Smart Speakern, spiegelt sich auch auf der Ebene des Forschungsdesigns wider. Somit trägt die Konstruktion von MMR-Designs, die sowohl Offline- als auch Online-Umgebungen berücksichtigen, nicht nur dazu bei, Validitätsbedrohungen zu minimieren, sondern auch den Aspekt der Gegenstandsangemessenheit aus medienpädagogischer Sicht abzusichern. Insbesondere mit Blick auf das wachsende Themenfeld 〈Big Data〉 bietet sich im MMR-Ansatz eine erweiterte 
Möglichkeit der Kreuzvalidierung zunehmend quantifizierter Analysen zu begegnen bei gleichzeitiger Stärkung qualitativer Analysen.

\section{Literatur}

Akremi, Leila. 2017. «Mixed-Methods-Sampling als Mittel zur Abgrenzung eines unscharfen und heterogenen Forschungsfeldes». Kölner Zeitschrift für Soziologie und Sozialpsychologie 69 (2): 261-86. https:// doi.org/10.1007/s11577-017-0460-3.

Baur, Nina, Udo Kelle, und Udo Kuckartz. 2017. «Mixed Methods - Stand der Debatte und aktuelle Problemlagen». Kölner Zeitschrift für Soziologie und Sozialpsychologie 69 (2): 1-37. https://doi.org/10.1007/s11577017-0450-5.

Burkart, Roland. 2002. Kommunikationswissenschaft: Grundlagen und Problemfelder. 4., überarb. und aktualisierte Aufl. Wien: Böhlau.

Castells, Manuel. 2017. Der Aufstieg der Netzwerkgesellschaft: Das Informationszeitalter, Wirtschaft, Gesellschaft, Kultur. 2. Aufl. Wiesbaden: Springer VS.

Creswell, John W., und Vicki L. Plano-Clark. 2018. Designing and Conducting Mixed Methods Research. 3. Aufl. Thousand Oaks: Sage.

Doering, Nicola, und Jürgen Bortz. 2016. Forschungsmethoden und Evaluation für Human- und Sozialwissenschaftler: Limitierte Sonderausgabe. 5. Aufl. Heidelberg: Springer-Verlag.

Fetters, Michael, und Dawn Freshwater. 2015. «The $1+1=3$ Integration Challenge». Journal of Mixed Methods Research 9 (2): 115-17.

Glantz, Alexander, und Tobias Michael. 2014. «Interviewereffekte». In Handbuch Methoden der empirischen Sozialforschung, herausgegeben von Nina Baur und Jörg Blasius, 313-22. Wiesbaden: Springer Fachmedien. https://doi.org/10.1007/978-3-531-18939-0_21.

Jahoda, Marie, Paul F. Lazarsfeld, und Hans Zeisel. 1975. Die Arbeitslosen von Marienthal. Ein soziographischer Versuch. Frankfurt am Main: Suhrkamp.

Johnson, R. Burke, und Larry Christensen. 2014. Educational research methods. Los Angeles: Sage.

Johnson, R. Burke, und Anthony J. Onwuegbuzie. 2004. «Mixed Methods Research: A Research Paradigm Whose Time Has Come». Educational Researcher 33 (7): 14-26. 
Johnson, R. Burke, Anthony J. Onwuegbuzie, und Lisa A. Turner. 2007. «Toward a Definition of Mixed Methods Research. Journal of Mixed Methods Research». Journal of Mixed Methods Research 1 (2): 112-33.

Jörissen, Benjamin, und Winfried Marotzki. 2009. Medienbildung - eine Einführung: Theorie - Methoden - Analysen. Bad Heilbrunn: Klinkhardt.

Kammerl, Rudolf. 2018. «Mediatisierung relationaler Ordnungen als Bedingung und Bezugspunkt von (Medien)Bildungsprozessen und (medien-) pädagogischer Theoriebildung». In Jahrbuch Medienpädagogik 14: Der digitale Raum - Medienpädagogische Untersuchungen und Perspektiven, herausgegeben von Manuela Pietraß, Johannes Fromme, Petra Grell, und Theo Hug. Wiesbaden: Springer Fachmedien. https:// doi.org/10.1007/978-3-658-19839-8_6.

Kuckartz, Udo. 2014. Mixed Methods: Methodologie, Forschungsdesigns und Analyseverfahren. Wiesbaden: VS Verlag für Sozialwissenschaften. https://doi.org/10.1007/978-3-531-93267-5.

Leech, Nancy L., und Anthony J. Onwuegbuzie. 2009. «A Typology of Mixed Methods Research Designs». Quality \& Quantity 43 (2): 265-75. https://doi.org/10.1007/s11135-007-9105-3.

Maxwell, Joseph A. 2013. Qualitative Research Design: An Interactive Approach. 3. Aufl. Thousand Oaks: Sage.

Nielsen, Jesper K. 2018. «Loudspeaker and Listening Position Estimation Using Smart Speakers». In 2018 IEEE International Conference on Acoustics, Speech and Signal Processing, 81-85. Calgary, Canada: IEEE Press.

Onwuegbuzie, Anthony J., und Kathleen M. T. Collins. 2017. «The Role of Sampling in Mixed Methods-Research». Kölner Zeitschrift Für Soziologie Und Sozialpsychologie 69 (2): 133-56. https://doi.org/10.1007/ s11577-017-0455-0.

Pietraß, Manuela. 2018. «Die Ermöglichung von Lernen und Bildung im digitalen Raum. Medienpädagogische Perspektiven». In Jahrbuch Medienpädagogik 14: Der digitale Raum - Medienpädagogische Untersuchungen und Perspektiven, herausgegeben von Manuela Pietraß, Johannes Fromme, Petra Grell, und Theo Hug, 11-32. Wiesbaden: Springer Fachmedien. https://doi.org/10.1007/978-3-658-19839-8_2.

Przyborski, Aglaja, und Monika Wohlrab-Sahr. 2014. Qualitative Sozialforschung: ein Arbeitsbuch. 4., erw. Aufl. München: Oldenbourg.

Pürer, Heinz, Nina Springer, und Wolfgang Eichhorn. 2015. Grundbegriffe der Kommunikationswissenschaft. Konstanz: UVK. 
RatSWD [Rat für Sozial- und Wirtschaftsdaten]. 2017. «Forschungsethische Grundsätze und Prüfverfahren in den Sozial- und Wirtschaftswissenschaften». RatSWD Output 9 (5). https://doi.org/10.17620/02671.1.

Schütz, Alfred. 1971. Das Problem der sozialen Wirklichkeit. Den Haag: Martinus Nijhoff.

Shannon, Claude E. 1948. «A Mathematical Theory of Communication». Bell System Technical Journal 27 (3): 379-423 und 623-56.

Tashakkori, Abbas, und Charles B. Teddlie. 1998. Mixed Methodology: Combining Qualitative and Quantitative Approaches. Thousand Oaks: Sage.

Teddlie, Charles B., und Abbas Tashakkori. 2009. Foundations of Mixed Methods Research: Integrating Quantitative and Qualitative Approaches in the Social and Behavioral Sciences. Thousand Oaks: Sage.

Wagner, Pia, und Linda Hering. 2014. «Online-Befragung». In Handbuch Methoden der empirischen Sozialforschung, herausgegeben von Nina Baur und Jörg Blasius, 661-73. Wiesbaden: Springer Fachmedien. https://doi.org/10.1007/978-3-531-18939-0_48.

Weaver, Warren, und Claude E. Shannon. 1949. A Mathematical Theory of Communication. Urbana: University of Illinois Press.

Welker, Martin. 2014. «Normalisierung und Ausdifferenzierung von Online-Forschung - Eine Einführung». In Handbuch Online-Forschung: Sozialwissenschaftliche Datengewinnung und -auswertung in digitalen Netzen, herausgegeben von Martin Welker, Monika Taddicken, JanHinrik Schmidt, und Nikolaus Jackob, 14-41. Köln: Herbert von Halem Verlag.

Zeller, Frauke. 2017. «Soziale Medien in der empirischen Forschung». In Handbuch Soziale Medien, herausgegeben von Jan-Hinrik Schmidt und Monika Taddicken, 389-408. Wiesbaden: Springer Fachmedien. https://doi.org/10.1007/978-3-658-03765-9_21. 